\title{
Recurrent Acute Erythroid Leukemia
}

National Cancer Institute

\section{Source}

National Cancer Institute. Recurrent Acute Erythroid Leukemia. NCI Thesaurus. Code C156723.

The reemergence of acute erythroid leukemia after a period of remission. 